\title{
Wave Structures in the Upper Ionospheric Plasma in Spherical Coordinates
}

\author{
Khatuna Elbakidze \\ Tbilisi State University, Tbilisi, Georgia \\ Email: khatuna.chargazia@gmail.com
}

How to cite this paper: Elbakidze, K. (2020) Wave Structures in the Upper Ionospheric Plasma in Spherical Coordinates. Journal of Applied Mathematics and Physics, 8, 1888-1895.

https://doi.org/10.4236/jamp.2020.89142

Received: July 1, 2020

Accepted: September 19, 2020

Published: September 22, 2020

Copyright $\odot 2020$ by author(s) and Scientific Research Publishing Inc. This work is licensed under the Creative Commons Attribution International License (CC BY 4.0).

http://creativecommons.org/licenses/by/4.0/

(c) (i) Open Access

\begin{abstract}
Physical mechanism of generation of the new modes of ultra-low-frequency (ULF) electromagnetic planetary waves in F-region of the spherical ionosphere due to the latitudinal inhomogeneity of the geomagnetic field is suggested. The frequency spectra, phase velocity, and wavelength of these perturbations are determined. It is established, that these perturbations are self-localized as nonlinear solitary vortex structures in the ionosphere and moving westward or eastward along the parallels with velocities much greater than the phase velocities of the linear waves. The properties of the wave structures under investigation are very similar to those of low-frequency perturbations observed experimentally in the ionosphere at middle latitudes.
\end{abstract}

\section{Keywords}

Ionospheric Plasma, Planetary Waves, Inhomogeneous Geomagnetic Field

\section{Introduction}

The increasing interest in large-scale (planetary) wave disturbances is caused by the fact that many ionospheric phenomena, such as super rotation of the Earth atmosphere [1], ionospheric precursors of natural processes [2] [3] and the ionospheric response to anthropogenic activity [4] [5] fall in the range of such waves. It was assumed that in natural conditions planetary waves generate in the ionosphere-stratosphere and propagate upward to ionospheric altitudes. However, the theoretical study of wave processes as the basis for energy transfers from lower to the upper atmosphere indicates that the system of stable zonal winds reliably screens (especially in summer) the upper atmosphere from the action of large-scale planetary waves generated in the troposphere-stratosphere [6]. Moreover, conditions most favorable for an upward propagation of only 
very long planetary waves (with a wavenumber of $1-2$ ) are created during equinoxes, when the zonal winds change their direction [7].

Nevertheless, a large body of observational data has been accumulated by now [8] [9] [10], which clearly indicate that fast large-scale electromagnetic disturbances are observed at the ionospheric regions [11] [12] [13]. It is also observed that the phase velocities of these disturbances differ by almost an order of magnitude at daily and night conditions. High phase velocities and their considerable variation from day to night make it impossible to identify these disturbances with magnetohydrodynamic waves.

Under natural conditions, these disturbances are observed as background oscillations. The observations indicate that induced oscillations of this type are generated at the impulse excitation of the ionosphere during magnetic storms [2] or under the action on the ionosphere from below as a result of earthquakes, volcanic eruptions or artificial explosions [3] [14] [15] in the latter case; the disturbances appear as solitary wave structure.

In this connection, it becomes extremely necessary to study whether planetary electromagnetic waves can be generated and changed in the ionosphere itself. It is necessary to determine the factors responsible for the generation of such disturbances to interpret adequately the processes proceeding in the ionosphere during wave formation and propagation.

\section{Initial Equations}

To study the generation of the large-scale low-frequency processes in the ionosphere the magneto-hydrodynamic equations are used for the ionosphere taking into account the fact, that large-scale flows do not perturb density and concentration of the medium particles [16]. The small-scale acoustic gravity and magnetohydrodynamic (Alfven, magnetosonic and helicon) waves are to be altered [17] [18]. The Coriolis force becomes a determining factor for atmospheric processes with a characteristic period of several hours and larger. As far as inhomogeneities of the Earth's rotation and the magnetic fields are permanently acting fundamental factors for the planetary-scale processes, it would be more appropriate to study the problem of generation of the planetary-scale ultra-lowfrequency (ULF) electromagnetic wave structures in $F$-layer of the ionosphere in a spherical coordinate system. Let $\theta$ denote an addition to the latitude $\varphi^{\prime}\left(\theta=\pi / 2-\varphi^{\prime}\right), \lambda$ is a longitude; $r$-distance from the earth center. The velocity component is assumed to be zero according to $r$ axis $-V_{r}=0 ; V_{\theta}(\theta, \lambda, t)$ is velocity component along meridian (it is positive if velocity is directed to the north). $V_{\lambda}(\theta, \lambda, t)$ is velocity component along the parallels (it is positive if velocity is directed to the east). For simplicity, the geomagnetic field is assumed to have only vertical component $H_{0 r}=-H_{p} \cos \theta$, i.e. the moderate and high latitudes are considered; where $H_{p}=5 \times 10^{4} \mathrm{nT}$ is the geomagnetic field strength near the pole. The angular rotational velocity component $\Omega_{r}=\Omega_{0} \cos \theta$ normal to the earth surface is important for the dynamical processes. 
The zonal wind determined by the experimental expression $\overline{V_{\lambda}}=\alpha r \sin \theta$, where $\alpha$ is a constant angular velocity of the atmosphere zonal circulation (so-called circulation index) [11] [12] [13]. The value of $\alpha$ varies seasonably: $\alpha=0.05 \Omega_{0}$-in winter, $\alpha=0.025 \Omega_{0}$-in summer. The stream function can be presented as $\Psi=\overline{\Psi(\theta)}+\Psi^{\prime}(\theta, \lambda, t)$. Then, ignoring $V_{r}$ in the continuity equation along with incompressibility term we determine the components of the velocity by stream function:

$$
\begin{gathered}
V_{\theta}=-\frac{1}{r \sin \theta} \frac{\partial \Psi}{\partial \lambda}, V_{\lambda}=\frac{1}{r} \frac{\partial \Psi}{\partial \theta}, \\
\overline{\Psi(\theta)}=-\alpha r^{2} \cos \theta .
\end{gathered}
$$

Taking into account above discussions and defined quantities, the close system of dynamical equations for the large-scale wave structures-the equation of motion of the medium particles and induction equation for dissipative ionosphere [16] can be reduced to the form:

$$
\begin{gathered}
\frac{\partial \Delta \Psi}{\partial t}+\alpha \frac{\partial \Delta \Psi}{\partial \lambda}+2\left(\alpha+\Omega_{0}\right) \frac{\partial \Psi}{\partial \lambda}+\alpha_{H} \frac{\partial h}{\partial \lambda}+\Lambda \Delta \Psi=-\frac{1}{R^{2} \sin \theta} J(\Psi, \Delta \Psi), \\
\frac{\partial h}{\partial t}-\Omega_{H} \frac{\partial \Psi}{\partial \lambda}+\alpha \frac{\partial h}{\partial \lambda}=-\frac{1}{R^{2} \sin \theta} J(\Psi, h) .
\end{gathered}
$$

Here, we introduce designations:

$$
\begin{aligned}
& \alpha_{H}=\frac{C_{H}}{R \sin \theta}, C_{H}=-\frac{c}{4 \pi e N} \frac{1}{R} \frac{\partial H_{0 r}}{\partial \theta}=-\frac{c H_{p}}{4 \pi e N R} \sin \theta, \\
& \Omega_{H}=\frac{N}{N_{n}} \frac{e}{M c \sin \theta} \frac{\partial H_{0 r}}{\partial \theta}=\frac{N}{N_{n}} \frac{e H_{p}}{M c}, h=\frac{N}{N_{n}} \frac{e R^{2}}{M c} h_{r}, \\
& \Delta=\frac{1}{\sin \theta}\left[\frac{\partial}{\partial \theta}\left(\sin \theta \frac{\partial}{\partial \theta}\right)+\frac{1}{\sin \theta} \frac{\partial^{2}}{\partial \theta^{2}}\right], J(a, b)=\frac{\partial a}{\partial \theta} \frac{\partial b}{\partial \lambda}-\frac{\partial a}{\partial \lambda} \frac{\partial b}{\partial \theta},
\end{aligned}
$$

where $N$ is a concentration of the charged particles; $N_{n}$-a neutral concentration; $M$ unit mass of ions and molecules; $c$ is a light speed; $R$-the earth radius, $e$-elementary charge. In our task $r$ is a parameter and therefore we shall replace $r$ by the earth radius $R$ in the (3)-(5) taking into account thickness of the atmosphere layer; $\Lambda$ is constant coefficient of surface friction between atmospheric layers and is of order of $10^{-5} \mathrm{~s}^{-1}$ for the ionospheric heights. Further for investigation the large-scale (planetary) zonal flows' dynamics in F-region of the ionosphere we will use the system of nonlinear Equations (3) and (4).

\section{Linear Approximation}

We begin an investigation of (3) and (4) from the analysis of motion at the initial stage when the amplitudes of the perturbations are small enough the nonlinear terms to be neglected in (3)-(4). Therefore, we shall seek the solution of these equations in the plane wave form $\Psi, h \sim f(\theta) \exp (i m \lambda-i \omega t)$ in linear approximation, where $m$ is a whole number; $\omega$-frequency of perturbations and $f(\theta)$ is an unknown function of $\theta$. 
Substituting the linear perturbed quantities into (3)-(4) we get a new equation for $f$.

$$
\begin{aligned}
& \frac{1}{\sin \theta} \frac{\partial}{\partial \theta}\left(\sin \theta \frac{\partial f}{\partial \theta}\right)+\left[-\frac{m^{2}}{\sin ^{2} \theta}+\frac{2\left(\alpha+\Omega_{0}\right) m}{\alpha m-\omega-i \Lambda}\right. \\
& \left.+\frac{\Omega_{H} \cdot m \cdot \alpha_{H} m}{(\alpha m-\omega)(\alpha m-\omega-i \Lambda)}\right] f=0
\end{aligned}
$$

The relation (6) presents the equation for the associated Lagrange polynoms. This equation has unique bounded solution if the sum of the second and third terms in the brackets equal to $n(n+1)$ (where $n$ is a whole number). The latter condition defines a dispersion equation:

$$
\frac{2\left(\alpha+\Omega_{0}\right) m}{\alpha m-\omega-i \Lambda}+\frac{\Omega_{H} \cdot m \cdot \alpha_{H} m}{(\alpha m-\omega)(\alpha m-\omega-i \Lambda)}=n(n+1),
$$

Submitting eigen-frequency $\omega_{0}$ and damping decrement $\gamma ; \omega=\omega_{0}+i \gamma$, $|\gamma| \ll \omega_{0}$, from (6) the dispersion relation for eigen frequencies:

$$
\omega_{0}^{2}-\omega_{0 p} \omega_{0}-\omega_{0 H} \alpha_{H} m=0,
$$

and expression for decrement:

$$
\gamma=-\frac{\omega_{0}^{\prime}}{2 \omega_{0}^{\prime}-\omega_{0 p}} \Lambda
$$

will be obtained, where $\omega_{0}^{\prime}=\alpha m-\omega_{0}, \omega_{0 p}=\frac{2\left(\alpha+\Omega_{0}\right) m}{n(n+1)}, \omega_{0 H}=\frac{\Omega_{H} m}{n(n+1)}$.

Let us estimate the order of the coefficients in Equation (8) for the characteristic values of the parameters in F-region of the ionosphere: $\Omega_{0}=7.3 \times 10^{-5} \mathrm{~s}^{-1}$, $\alpha=0.04 \Omega_{0} \sim 3 \times 10^{-6} \mathrm{~s}^{-1}, \quad H_{p}=5 \times 10^{-5} \mathrm{~T}, \quad N_{n}=3 \times 10^{14} \mathrm{~m}^{-3}, \quad N=3 \times 10^{11} \mathrm{~m}^{-3}$, $R=6.4 \times 10^{6} \mathrm{~m}$. Thus, $\alpha_{H}=10^{-3} \div 10^{-4} \mathrm{~s}^{-1}, 2 \Omega_{H} \approx 10^{-4} \div 10^{-5} \mathrm{~s}^{-1}, \Lambda=10^{-5} \mathrm{~s}^{-1}$. It follows, that $\alpha_{H} m \gg \omega_{0 p}, \Lambda, \omega_{0 H}, \alpha m, \omega_{0}^{\prime} \approx \omega_{0}$. Taking all these into account, from (8) we get a spectrum of oscillations:

$$
\omega_{0}= \pm \frac{H_{p} m}{\sqrt{4 \pi \rho n(n+1)} R}, \quad \gamma=-\frac{1}{2} \Lambda .
$$

We introduce the angular velocity of the wave propagation $\mathrm{d} \sigma / \mathrm{d} t=\omega_{0} / \mathrm{m}$, using (10) and determine the linear phase velocity of the wave motion along the latitudinal circles (parallels) $-V_{\lambda}=V_{p h}=R \sin \theta \cdot \mathrm{d} \sigma / \mathrm{d} t$. Thus, we get the expression for the phase velocity of the waves:

$$
V_{p h}= \pm \frac{H_{p}}{\sqrt{4 \pi \rho n(n+1)}} \sin \theta
$$

This wave is the fast and represents itself a new mode of eigen-frequencies of the oscillations in F-region of the ionosphere. The wave (10), (11) is weakly damped with decrement: $\gamma=10^{-6} \mathrm{~s}^{-1}$ and can propagate along the parallel to the west as well as to the east. The wavelength is $10^{3} \mathrm{~km}$ or great.

From induction Equation (4) amplitude of the geomagnetic pulsations gener- 
ated by these waves, $h_{r} \approx H_{0 r} \xi / R$, where $\xi$ is a transversal displacement of the charged particles, can be estimated. At displacement $\xi$ equals 0.1 and $1 \mathrm{~km}$ for $\theta=45^{\circ}, h_{r}$ is equal to 8 and $80 \mathrm{nT}$ respectively.

\section{Nonlinear Evolution of the Initial Equations}

Investigating the system (3)-(4) at the further stage, when the amplitudes of the perturbations are sufficiently grown, the nonlinearity becomes important In determining the dynamical processes. So, for further analysis of the ionospheric energy transfer processes the close system of nonlinear Equations ((3), (4)) should be considered. For simplicity we consider the motion at any fixed latitude $\varphi_{0}=\pi / 2-\theta_{0}$. It is convenient to introduce the new latitudinal and longitudinal coordinates $x=\lambda R \sin \theta_{0}, \quad y=-\left(\theta-\theta_{0}\right) R$, the latter transformation causes the freezing of variable coefficients in the system of Equations (3), (4) in non-dissipative approximation $(\Lambda=0)$. We get a closed system of nonlinear equations with constant coefficients solution of which seek in a form of the stationary regular waves $\Psi=\Psi(\eta, y), h=h(\eta, y)$, spreading along the latitudes $x$ with velocity $U$, without changing the shape, $\eta=x-U t \quad[18]$ :

$$
\Delta_{\perp} \Psi-\beta_{2} y=F\left(\Psi+\beta_{1} y\right)
$$

where $F$ is an arbitrary chosen differential function of its argument,

$$
\beta_{1}=C_{R}-U, \beta_{2}=\frac{2\left(\alpha+\Omega_{0}\right)+C_{H} \Omega_{H}}{R\left(C_{R}-U\right)} \sin \theta_{0},
$$

where $C_{R}=R \sin \theta_{0}$ is the characteristic phase velocity of ordinary Rossby waves.

Following [19] we use the polar coordinates along the earth surface $r=\left(\eta^{2}+y^{2}\right)^{1 / 2}, \operatorname{tg} \varphi=y / \eta$ and the circle with the radius a. Further, the quantities $\Psi(r, \varphi)$ and $h(r, \varphi)$ is requested to be twice continuously differentiable (including the circle $r=a$ ) along their arguments and to vanish exponentially at $r \rightarrow \infty$. Then (12) will have the following solutions:

$$
\begin{gathered}
\Psi(r, \varphi, t)=-\frac{C_{R}-U}{\Omega_{H} R \sin \theta_{0}} h(r, \varphi, t)=a \beta_{1} F(r) \sin \varphi, \\
F(r, t)=\left\{\begin{array}{l}
-(p / \chi)^{2} J_{1}(\chi r) / J_{1}(\chi a)-\left(\chi^{2}+p^{2}\right) r /\left(a \chi^{2}\right), r<a, \\
-K_{1}(p r) / K_{1}(p a), r \geq a ;
\end{array}\right.
\end{gathered}
$$

where $J_{n}$ is nth degree Bessel function and $K_{n}-$ McDonald function; $p$ and $\chi$ parameters are related by the dispersion relation:

$$
\frac{J_{1}(\chi a)}{\chi J_{1}(\chi a)}=-\frac{K_{1}(p a)}{p K_{2}(p a)}, \quad p^{2}=\frac{\beta_{2}}{\beta_{1}}>0,
$$

According the dispersion Equation (16), solution (14) has two free parameters $U$ and $a$. The disturbed solution obtained from (13) equals to $\Psi, h \sim r^{-1 / 2} \exp (-p r)$ at $r \rightarrow \infty$. This indicates that the wave is localized on the earth surface $(\eta, y)$. This type structures represent a pair of oppositely circulating vortices (cyc- 
lone-anticyclone) of equal intensity, spreading along the parallels on the background of purely zonal mean west to east flow. The nonlinear vortex structures move with velocity $U>C_{R}-C_{H} \Omega_{H} /\left(2 \alpha+2 \Omega_{0}\right)$. The characteristic scale is $d \sim a \approx\left[U R /\left(\alpha+\Omega_{0}\right)\right]^{1 / 2} \sim 10^{4} \mathrm{~km}$.

The above wave structures will be generated first at the action on the ionosphere from above (e.g. by geomagnetic sub storms) or from below (by earthquakes, major artificial explosions, etc.).

\section{Discussion and Conclusions}

It has been established that large-scale (planetary) linear and nonlinear electromagnetic wave structures, caused by the permanently acting factor (latitude variation in the geomagnetic field), can be generated in the ionospheric dynamo region. Considering large-scale ionospheric flows with the characteristic extend of $10^{3} \mathrm{~km}$ and larger, we cannot ignore nonuniformity of the geomagnetic field and the Earth rotation angular velocity, since the ordinary Rossby parameter $\beta=\partial 2 \Omega_{0 z} / \partial y$ and its magnetic analog $\beta_{H}=e N\left(\partial H_{0} / \partial y\right) / \rho c$ are of the same order of magnitude in the $F$-region: $\beta \cong 1.3 \times 10^{-11} \mathrm{~m}^{-1} \cdot \mathrm{s}^{-1}$,

$\beta_{H} \cong 5.4 \times 10^{-11} \mathrm{~m}^{-1} \cdot \mathrm{s}^{-1}$. The waves have a frequency of the order of $\left(10^{-1}-10^{-6}\right) \mathrm{s}^{-1}$ and a wavelength of about $10^{3} \mathrm{~km}$ and longer and are almost nondamped $\gamma=10^{-6} \mathrm{~s}^{-1}$. According to the Maxwell equation for the current $j^{+}=e N V_{D}$, fast waves generate substantial micro pulsations of the geomagnetic field, which's order of magnitude (where $\lambda^{+} \sim 10^{3} \mathrm{~km}$ is the wavelength of fast waves). Thus, $h_{z}^{+} \sim 10^{3} \mathrm{nT}$.

Based on the analytical solutions of the system of nonlinear dynamic Equations (3), (4), it has been shown that the large-scale electromagnetic waves are self-localized in the nondissipative ionospheric $F$-region in the form of nonlinear solitary vortex structures moving at the constant velocity along latitudinal circles. Nonlinear structures are composed of the pair of oppositely rotating dipole vortices (cyclone-anticyclone) and carry entrapped particles of the medium. The electromagnetic perturbations of slow $d^{-}$and fast $d^{+}$vortices are not less than $10^{3}$ and $10^{4} \mathrm{~km}$ respectively.

According to the equations of transport (3), (47) in the dissipative ionosphere, the energy and enstrophy of large-scale vortices are much greater than the dissipative term, and, correspondingly, such vortices relax very slowly.

Thus, nonlinear large-scale electromagnetic vortex structures are long-lived formations in the ionospheric dynamo region and, therefore, can play a substantial role in the processes of matter, heat and energy transfer and in strong turbulation of the medium [20].

The considered wave structures are the natural degrees of freedom of the ionospheric $F$-region. Therefore, if the ionosphere is affected from above or below magnetic storms, earthquakes, artificial explosions, etc., the wave structures will first of all be generated and/or enhanced on these models [21]. Solitary vortices will be generated at a certain power of a source [18], which is corroborated by 
observations [4] [22] [23].

\section{Acknowledgements}

The research leading to these results has received funding from the Shota Rustaveli National Science Foundation project N FR17_279.

\section{Conflicts of Interest}

The author declares no conflicts of interest regarding the publication of this paper.

\section{References}

[1] Kazimirovski, E.S. and Kokourov, V.D. (1979) Motions in the Ionosphere. Nauka, Novosibirsk, 344.

[2] Haykowicz, L.A. (1991) Global Onset and Propagation of Large-Scale Travelling Ionospheric Disturbances as a Result of the Great Storm of 13 March 1989. Planetary and Space Science, 39, 583-593. https://doi.org/10.1016/0032-0633(91)90053-D

[3] Liperovski, V.A., Pokhotelov, O.A. and Shalimov, S.A. (1992) Ionospheric Precursors of Earthquakes. Nauka, M., 304.

[4] Shaefer, L.D., Rock, D.R., Lewis, J.P., et al. (1999) Detection of Explosive Events by Monitoring Acoustically-Induced Geomagnetic Pertubations. Lawrence Livermore Laboratory, Livermore, 63.

[5] Pokhotelov, O.A., et al. (1999) Modification of the Ionosphere during Hostilities in Zone of the Persian Golf. Doklady Akademii Nauk SSSR, 321, 1168-1172.

[6] Khantadze, A.G. and Sharadze, Z.S. (1980) Ionospheric Effects of Planetary Waves in Wave Pertubation in the Atmosphere. Nauka, Almaata, 143.

[7] Khantadze, A.G., Sharadze, Z.S. and Kobaladze, Z.L. (1988) On the Alfen-Type Planetary Waves in the Ionosphere, in Investigation of Dynamical Processes in the Upper Atmosphere. Gidrometeoizdat, M., 110.

[8] Sharadze, Z.S. and Khantadze, A.G. (1979) Planetary Waves in the E- and F-Region of the Ionosphere. Soobscenija Akademii Nauk Gruzinskoj SSR, 94, 69-72.

[9] Sharadze, Z.S. (1979) Atmospheric Waves in the Ionosphere during Winter StratosphericWarmings. Ionosfernie Issledovania, No. 29, 28-39.

[10] Aroshidze, G.M., Kikvilashvili, G.B., Liadze, Z.L., et al. (1977) Midlatitude Es Layer and Wave Pertubations in the Ionospheric E-Region. Ionosfernie Issledovania, No. $24,82-86$.

[11] Sharadze, Z.S., et al. (1991) Phenomena in the Midlatitude Ionosphere Associated with Atmospheric Waves. Doctoral Dissertation, IZMIRAN, Moscow, 255 p.

[12] Sharadze, Z.S. (1989) Major Earthquakes and Associated Disturbances in the Ionosphere and Geomagnetic Field. Fizika Zemli, No. 1, 20-31.

[13] Sharadze, Z.S., et al. (1988) Wave Pertubations of Nonacoustic Origin in the Midlatitude Ionosphere. Geomagnetizm i Aeronomia, 28, 446-451.

[14] Cheng, K. and Huang, Y.-N. (1992) Ionospheric Disturbances Observed during the Period of Mount Pinatubo Eruption in June 1991. Journal of Geophysical Research, 97, 16995-17004. https://doi.org/10.1029/92JA01462

[15] Drobzhev, V.I., Moloetov, G.F., Sharadze, Z.S., et al. (1986) Ionospheric Response to the Disturbances Induced by an Industrial Explosions. Ionosfernie Issledovania, 
No. 39, 61-76

[16] Chargazia, Kh. and Kharshiladze, O. (2014) Weather Forming Ultra Low Frequency Electromagnetic Waves at Interaction with Local Inhomogeneous Winds in the Ionosphere. Sun and Geosphere.

[17] Khantadze, A.G. (1973) Some Problems in Dynamics of the Conducting Atmosphere. Nauka, Tbilisi, 279 p.

[18] Gossard, E. and Hook, W. (1975) Waves in Atmosphere. Elsevier, Amsterdam, 532 p.

[19] Aburjania, G. (1996) Self-Organization of Acoustic-Gravity Vortices in the Ionosphere before Earthquake. Plasma Physics Report, 22, 954-959.

[20] Petviashvili, V.I. and Pokhotelov, O.A. (1989) Solitary Waves in Plasma and Atmosphere. Enargoatomizdat, M., 199 p.

[21] Aburjania, G.D. (1996) Self-Organization of Acoustic-Gravity Vortices in the Ionosphere Aprior to an Earthquake. Fizika Plazmy, 22, 954-959.

[22] Aburjania, G.D. (1990) Structural Turbulence and Diffusion of Plasma in Magnetic Traps. Fizika Plazmy, 16, 70-76.

[23] Aburjania, G.D. and Machabeli, G.Z. (1998) Generation of Electromagnetic Perturbations by Acoustic Waves in the Ionosphere. Journal of Geophysical Research, 103, 9441-9447. https://doi.org/10.1029/97JA03060 